\title{
Die Manifestierung von Geschlechterstereotypen in Metaphern der deutschen Jugendsprache
}

\author{
Oksana Khrystenko (Sumy)
}

\begin{abstract}
The article considers gender-based substandard metaphors with stereotypical background in the German youth language. The aim of the publication is to highlight the peculiarities in metaphorical representation of males/females relating to their appearance, character, behavioral and intellectual characteristics. The substandard metaphors with a reference to male/female correlate with the conceptual metaphors grounded in sensory-motor experience. It can be concluded that the metaphors in the youth language verbalize gender stereotypes existing in society. The substandard metaphors that refer to persons of both sexes challenging traditional gender stereotypes and falling into the category of 'deviant other' have negative connotation.
\end{abstract}

\section{$1 \quad$ Einleitung}

Die anthropozentrische Ausrichtung und Interdisziplinarität der modernen Sprachwissenschaft setzen voraus, dass immer mehr Forscher ihre Aufmerksamkeit auf die Frage der sprachlichen Manifestierung von Strukturen der Wissensrepräsentation richten. Einen bedeutsamen Platz in diesem Forschungsparadigma nimmt die Untersuchung der Spezifik verbaler Objektivierung von stereotypen Vorstellungen ein (Allport 1971; Quasthoff 1973; Zybatow 1995; Bartminski 2005). Trotz der Vielzahl an Studien zu diesem Thema besteht eine gewisse Uneinigkeit unter den Linguisten, was die Fragen der Typologie, des Bedeutungsumfangs und der sprachlichen Manifestierung von Stereotypen angeht, was auch durch die Schwierigkeit bei deren Erfassung bedingt ist.

Die Geschlechterstereotype haben die Aufmerksamkeit sowohl in sozial-psychologischen, als auch in linguistisch ausgerichteten Forschungen gefunden. Ein Zeugnis dafür sind vor allem die vorhandenen sozial-psychologischen und sprachwissenschaftlichen Arbeiten (Eckes 2010; Eckert/McConnell-Ginet 2006; Alfermann 1996; Basow 1992). Trotz dieser Untersuchungen stellt die Erforschung der Geschlechterstereotype bei deutschen Jugendlichen ein Forschungsdesiderat dar. Dass die Geschlechterdimension in der Jugendforschung generell noch wenig ausgearbeitet ist, hat H.-M. Trautner konstatiert: „Wenn man nach dem Stellenwert des Jugendalters in der Geschlechterforschung fragt, so findet man, dass das Jugendalter gegenüber der Kindheit und auch gegenüber dem Erwachsenenalter eher vernachlässigt wird“" (Trautner 2007: 31). 
In die Zielsetzung dieses Beitrags gehen folgende Fragen ein:

1. Welche Geschlechterstereotype kommen in metaphorischer Form zum Ausdruck?

2. Gibt es wesentliche Unterschiede in der Repräsentation der Frau/des Mannes in der Substandardmetapher ${ }^{1}$ ?

3. Welche Metapherngruppen - nach der Gemeinsamkeit ihres Motivationsmusters ${ }^{2}$ - haben die Geschlechterstereotype als Basis? Sind diese Metapherngruppen mit metaphorischen konzeptuellen Modellen verbunden?

\section{Zum Stereotypenbegriff}

Der Begriff Stereotyp wurde zunächst in die Sozialwissenschaften im weiten Sinne als Bewusstseinsbild, das die öffentliche Meinung repräsentiert, aufgenommen. Diese Ansicht war vor allem in den Werken von W. Lippmann (1990: 28) vertreten, der die Stereotype als „Bilder in den Köpfen menschlicher Wesen, die Bilder von sich selbst, von den anderen, von ihren Bedürfnissen, Zielen und Beziehungen zueinander" interpretiert hat. Diese vereinfachten Bilder resultieren aus unserer unmittelbaren Welterkenntnis und stellen hiermit „kognitive Schematisierungen“ (Konerding 2001: 152) dar. Im Allgemeinen sind bei der Stereotypisierung einige wichtige Prinzipien entscheidend - v. a. das „Energiesparen“ (McGarty/Yzerbyt/Spears 2002: 2), wenn Erscheinungen und Objekte unbewusst in eine Kategorie eingeordnet werden. Andererseits werden die Stereotype als „Marker der sozialen Organisation“ (ibd: 12) bezeichnet, weil sie die sozial geteilten Überzeugungen über Eigenschaften einer Person bzw. Verhaltensweisen einer menschlichen Gruppe vermitteln. Außerdem wird dem Stereotyp wie jeder Überzeugung eine gewisse Schnelllebigkeit, da es dem Individuum ermöglicht, sich ,der jeweiligen Stimmung und den Bedürfnissen der Situation anzupassen“ (Allport 1971: 212). Die Multidimensionalität des Stereotyps erlaubt seine Interpretation als soziales, kulturelles und kognitives Phänomen.

Als soziales Phänomen vermittelt das Stereotyp bestimmte, innerhalb einer sozialen/ethnischen Gruppe erworbene, Erfahrung, die als Resultat der kollektiven Welterkenntnis zu interpretieren ist. Gleichzeitig gehört „der Stereotypeninhalt der sozialen Wirklichkeit, d. h. den Menschen als Vertreter eines Volkes, einer Klasse, eines Milieus“ (Bartminski 2005: 142). So ist die Entwicklung von oft positiv und negativ beladenen Autound Heterostereotypen als ,vereinfachte und typisierte Vorstellungen darüber, welche Eigenschaften bzw. Verhaltensweisen Vertreter einzelner sozialer/ethnischer Gruppen besitzen“ (cf. Schmidt 2002: 291) innerhalb des sozialen Kontextes erklärbar.

Zugleich bedeutet die Zugehörigkeit zu einer ethnischen und sozialen Menschengruppe auch die Zugehörigkeit zu einer Kultur bzw. Subkultur mit ihren Werten und Orientierungen, was die kulturelle Determiniertheit der stereotypen Vorstellungen voraussetzt. Die „sprachlich-

\footnotetext{
${ }^{1}$ Unter der Substandardmetapher wird hier die Bedeutungsübertragung im sprachlichen Substandard verstanden, der den Gesamtbereich der Sprachvarietäten unterhalb des Standards umfasst.

2 Die Metaphernklassifikation nach der Gemeinsamkeit des Motivationsmusters umfasst die bedeutungsunterschiedlichen Metaphern, deren Referent zu einer thematischen Sphäre gehört (Essen, Tier, Mensch, Politik, Pflanze usw.).
} 

deutschen Jugendsprache

kulturelle Prägung bestimmt somit unsere Erwartungen, Vorlieben, Vorurteile und Vorstellungen von uns selbst und von anderen und bildet somit die Grundlage für die Begegnung und Interaktion mit anderen Kulturen“" (Kilian 2015: 157).

Aus Sicht der kognitiven Linguistik hängt der Prozess der Stereotypenbildung mit der Fähigkeit des menschlichen Bewusstseins zusammen, die Information über ähnliche Erscheinungen, Fakten zu verallgemeinern und zu behalten. Alle diese Prozesse sind mit der Erkenntnis der Welt und der „Verarbeitung von Informationen verschiedener Art über Personen, Gruppen, Situationen“ (Eckes 1997: 18) verbunden. Die kognitive Präsentation von Stereotypen ist aber unzertrennlich mit deren sozialer Natur verbunden - ,prejudice is both cognitive and social phenomenon. It isn't merely a characteristic of individual beliefs or emotion about social group, but a shared form of social representation of group members"3 (van Dijk 1984: 13).

In den sprachwissenschaftlichen Forschungsarbeiten der letzten Jahre ist eine eher breite Auffassung des Stereotyps als einer im kollektiven Bewusstsein verankerte Vorstellung vertreten (Putnam 1979; van Dijk 1984; Bartminski 2005). Die vorgestellte Forschungsperspektive schließt sich der verbreiteten Auffassung des Stereotyps an: als eine vereinfachte kulturdeterminierte Vorstellung über Objekte der Wirklichkeit, die aus menschlicher Welterkenntnis und der Sozialisationserfahrung innerhalb einer Menschengruppe resultiert, und sich in der Sprache manifestieren kann.

\section{Die sprachlichen Ebenen des Stereotypenausdrucks}

Die meisten Stereotypenklassifikationen berücksichtigen den Einfluss sozialanthropologischer Faktoren auf die Herausbildung von stereotypen Vorstellungen (Alter, Geschlecht, Ethinizität- und Kulturzugehörigkeit, sozialer Status) und unterscheiden die sprachlichen Ebenen des Stereotypenausdrucks.

Was die sprachliche und außersprachliche Manifestierung von Stereotypen angeht, werden in der Sprachwissenschaft verschiedene Ansichten unterschieden:

- $\quad$ die Möglichkeit des non-verbalen Ausdrucks von Stereotypen wird bestritten. Der Ansatz wird hauptsächlich in den Werken von Adam Schaff (Schaff 1979/1980) vertreten, der die ausschließliche Präsenz des Stereotyps in der Form des Wortes bzw. einer Aussage betont (,uns genügt die Feststellung der Tatsache, dass die Stereotypen immer verbalen Charakter haben, d.h., dass sie immer in Verbindung mit einem bestimmten Wort oder Ausdruck auftreten“" (Schaff 1980: 36);

- die Existenz von non-verbalen (Denkstereotypen) und verbalen Stereotypen (Stereotype, für die eine bestimmte sprachliche Formulierung festgelegt ist) wird anerkannt (Gülich 1978). Es lässt sich sagen, dass der Zusammenhang von Denk- und Sprachstereotypen

\footnotetext{
${ }^{3}$ In seiner Arbeit gebraucht Teun van Dijk Termini prejudice-stereotypes als synonymisch „after a brief summary of classical and more recent approaches to ethnic attitudes, stereotypes or prejudice, we present our own theoretical framework“" (van Dijk 1984: 13).
} 
unmittelbar ist, weil Stereotypen in erster Linie kognitive Strukturen (cf. Eckes 1997:17) sind, die sich dann in der Sprache manifestieren können.

Was das Kriterium des Ausdrucks von Stereotypen in der Sprache angeht, unterscheidet die Mehrheit der Sprachforscher zwischen einer Stereotypenmanifestierung auf dem Satz- oder auf dem Wortniveau. Die deutsche Sprachwissenschaftlerin U. Quasthoff (1973) unterscheidet auf dem Satzniveau vier Stereotypenarten:

- Stereotype, die eine logische Struktur F (x) haben, die ihrerseits in der sprachlichen Form des Typs (der Deutsche ist fleißig- $[D(x) \rightarrow F(x)]$ ) zum Ausdruck kommt. Die Eigenschaft, die in dem Satztyp einzelnen Vertretern einer ethnischen/sozialen Gruppe zugeschrieben wird, wird in der Regel auf andere Vertreter dieser Gruppe übertragen (Für alle $x$ gilt, wenn $x$ ein Deutscher ist, dann gehört er zur Klasse der Fleißigen);

- Stereotype, die in Sätzen wie „die Angehörigen einer Gruppe gelten als x“ zum Ausdruck kommen, was die Satzstruktur „die Angehörigen einer Gruppe sind x" (Der niedersächsische Mensch gilt als wortkarg $\rightarrow$ die Niedersachsen sind wortkarg) präsuppositioniert;

- Satzstereotype, in denen der Sprecher eine eigene Einstellung zu den Vertretern einer bestimmten ethnischen/sozialen Gruppe ausdrückt (ich glaube, dass die Amerikaner...). Die Aussagen haben eine entsprechende Präsupposition (Amerikaner sind...);

- „Textlinguistischer Typ“ von Stereotypen (cf. Quasthoff 1973: 254), der mit der Kategorie subjektiver Modalität verbunden ist (bsw. der Gebrauch der adversativen Konjunktion aber: „Er ist Jude, aber sehr nett“, implizit wird hierbei ausgedrückt, dass die Juden eigentlich nicht nett sind).

Die Möglichkeit der lexikalischen Manifestierung von Stereotypen hängt im Wesentlichen vom Verständnis der konnotativen Komponente der Wortsemantik ab. Wenn die Konnotation im engen Sinne nur als emotive und stilistische Färbung der lexikalischen Einheit verstanden wird, sind die Konnektionen zwischen dem Stereotyp und der lexikalischen Bedeutung schwer zu überprüfen - „die mit dem Wort Nigger verbundenen Konnotationen sind lediglich negativ wertend, sie sagen nichts Inhaltliches über den Charakter der entsprechenden Gruppe aus“" (Zybatow 1995: 26).

Eine weit ausgelegte Auffassung der Konnotation in der Sprachwissenschaft betrachtet diese als Makrokomponente der lexikalischen Bedeutung, die sog. semantische Assoziation ${ }^{4}$, die durch die in der Gesellschaftspraxis existierenden kulturellen Vorstellungen und Traditionen determiniert ist. Solch eine Auffassung des Begriffs 'Konnotation' ermöglicht die Behauptung, dass sich Stereotype in der Wortsemantik widerspiegeln.

Nach H. Putnam benötigen die stereotypen Vorstellungen bestimmte Assoziationsnennungen, dass ,z. B. Tiger Großkatzen sind, wie groß sie sind, Fellfarbe (schwarz gestreift), dass sie wild sind und im Dschungel leben“ (Putnam 1979: 71). Daher wird die Bedeutung des Wortes vom Forscher als ein Vektor beschrieben. Außer den syntaktischen und semantischen Markern in der Wortbedeutung (Wasser - Substantiv, konkret; natürliche Art, Flüssigkeit)

\footnotetext{
${ }^{4}$ Der Terminus „semantische Assoziation“ ist in den Arbeiten von J. Apresian (Apresian 1995: 67) vorhanden.
} 
deutschen Jugendsprache

werden von H. Putnam zusätzliche Komponenten herausgesondert (farblos, durchsichtig, ohne Geschmack, durstlöschend), die „konventionell verwurzelte Meinungen darüber vertreten, wie ein x aussehe, was er tue oder sei“ (Putnam 1979: 68). Obwohl die Putnamsche Stereotypvorstellung oft aufgrund der zu wenig ausgeprägten Unterscheidungsmerkmale von der Merkmalssemantik kritisiert wird ${ }^{5}$, wird das auf der Wortebene manifestierte Stereotyp als Komponentenstruktur angesehen, die aus den Erkenntnis- und Bewertungskomponenten besteht (cf. Bartminski 2005). Wenn die Erkenntniskomponenten im Stereotyp auf der objektiven Welterkenntnis und realen Fakten beruhen, sind die Bewertungskomponenten sekundär und können dem Lexem emotionale Schattierung verleihen (ibd.: 167). So sind bspw. in der deutschen Umgangssprache manche Bezeichnungen von Vertretern einzelner Berufe negativ markiert („Bauer“ symbolisiert Begrenztheit). Von der Komponentenstruktur des Stereotyps ausgehend unterscheidet der polnische Wissenschaftler Jerzy Bartminski (Bartminski 2005: 167) folgende Varianten der stereotypen Vorstellungen - solche, die reale Eigenschaften der Erkenntnisobjekte widerspiegeln (so, wie es ist) und stereotypische Muster, die, was die Normen angeht, als notwendig verstanden werden und in der Formel (solcher, wie er sein muss) vereinigt werden können6 ${ }^{6}$. In der Wortsemantik können sich aber die assoziativen Vorstellungen über ein ideales Objekt/Subjekt (wie es sein muss) und die Vorstellungen über die realen Eigenschaften eines Erkenntnisobjektes (wie es ist) überschneiden, auch können Stereotype nach dem Muster (wie es sein muss) oft die Vorstellungen über Realität determinieren. So können die stereotypischen in einer Bedeutungsübertragung zustande kommenden Vorstellungen darüber, wie eine Frau/ein Mann aussehen muss, von existierenden Idealen determiniert werden. Die Entsprechung der idealen Vorstellungen über eine Frau/einen Mann führt oft dazu, dass die herabgesetzten Lexeme positiv konnotiert sind.

\section{Geschlechterstereotype}

\subsection{Theorien zur Entstehung von Geschlechterstereotypen}

Die Theorien der Entstehung von Geschlechterstereotypen sind mehrfach in sozial- und sprachwissenschaftlichen Arbeiten erörtert worden7 ${ }^{7}$ In diesem Aufsatz werden nun die wichtigsten Theorien skizziert.

Die meisten existierenden Theorien und Hypothesen, die auch die Entstehung von Geschlechterstereotypen als soziokognitives Phänomen begründen, können in soziale Lerntheorien und kognitive Theorien eingeteilt werden. Die sozialen Lerntheorien gehen von dem Begriff geschlechtsspezifische Sozialisation aus. Demnach erfolgen die Übernahme von Geschlechtsrollen und die Herausbildung der Geschlechtsidentität durch Nachahmung von

\footnotetext{
${ }^{5}$ In der Klassifikation von H. Putnam „wurden die typischen unmittelbaren Bedeutungskomponenten den Stereotypen angeglichen“ (Busse 2009: 60). Nach Schmidt (2002: 293) sollte der Unterschied in der oben erwähnten Klassifikation in syntaktische und semantische Merkmale zweifelhaft sein.

${ }^{6}$ Außerdem werden von J. Bartminski (2005) mythologische (Vorstellungen von Märchen/ Mythosgestalten) und ideologische (Vorstellungen von sozialen Situationen oder abstrakte Begriffe) Stereotype unterschieden.

${ }^{7}$ Eckes 2004; Allport 1971; Basow 1992; Alfermann 1996.
} 
entsprechenden Verhaltensweisen und Orientierung an äußerlich sichtbaren Merkmalen (cf. Bandura/Bussey 1984: 1292). Die Fähigkeit zwischen Geschlechtern differenzieren und sich selbst mit dem Geschlecht identifizieren zu können ${ }^{8}$ wird von den Anhängern dieser Theorie „auf die geschlechtsbezogenen Erziehungspraktiken und Verhaltensdifferenzierungen der sozialen Umwelt zurückgeführt“ (Trautner 1997: 408). Die geschlechtsspezifische primäre Sozialisation im Kinderalter schließt die Annahme über Geschlechtersegregation der Kinder in den geschlechtshomogenen Spiel- und Freundschaftsgruppen ein, in welchen verschiedene Normen, Verhaltensmuster und als Resultat ein unterschiedliches Weltverständnis entwickelt werden (cf. Eckert/McConnell-Ginet 2006: 24). Die geschlechtshomogenen kleinen Freundschaftsgruppen des Kinderalters werden im Adoleszenzalter in „die führende Gruppe ${ }^{9}$ integriert, welche infolge der neuen Interaktion zwischen Mädchen und Jungen entsteht" (Eckert 2014: 251). Diese führende Jugendgruppe, die die anderen aus den Normen der Kinderwelt herausführt, ist der Wegbereiter für die adoleszenten Praktiken, welche mit dem Autoritätsgewinn innerhalb der Gruppe verbunden sind. Die Autorität innerhalb der Jugendgruppe kann laut Penelope Eckert (2014: 252) dank der heterosexuellen Erwünschtheit, Etablierung bestimmter Aktivitätsstile und Sprachpraktiken gewonnen werden. In der Adoleszenz „transformieren sich auch die Geschlechterentwürfe und die herrschenden Geschlechternormen werden variiert" (King 2009: 12). Die Variation betrifft z.T. auch die Geschlechterstereotype, die von der Übernahme neuer sozialer Rollen beeinflusst werden. So ,treten mit der Zunahme gegengeschlechtlicher Interaktion im Jugendalter neben die überwiegend negativ gefärbten Charakterisierungen des anderen Geschlechts nach und nach positive Merkmale“ (Eckes 2010: 181).

Die kognitiven Theorien gehen von der menschlichen Fähigkeit aus, Geschlechter zu kategorisieren. Laut diesen Theorien ,agieren die Menschen als Konstrukteure ihrer Umwelt und dadurch nehmen sie wesentlich Einfluss auf ihre eigene Entwicklung" (Alfermann 1996: 62-63). Demnach vollzieht sich die Geschlechtstypisierung als „Entwicklungsprozess der Wahrnehmung und Verarbeitung der Geschlechterdifferenzierung in der sozialen Umwelt" (Trautner 1997: 409) nicht nur durch Erkennen der eigenen Geschlechtsidentität und Geschlechtsidentifikation, sondern auch durch die Ausbildung der geschlechtsbezogenen Einstellungen. „Der entscheidende Punkt der kognitiven Entwicklungstheorie ist somit, dass mit dem Erwerb der Geschlechteridentität mehr oder weniger automatisch damit konsistente Einstellungen und Verhaltensweisen erworben werden. Dies soll v. a. durch Nachahmung gleichgeschlechtlicher Modelle geschehen, weil diese der eigenen Geschlechtsidentität entsprechen“(ibd: 71). Die Geschlechtstypisierung schafft damit eine Grundlage für die Herausbildung bestimmter Geschlechtervorstellungen, welche dann als Beurteilungen auf Mitglieder anderer sozialer Gruppen angewendet werden können.

\footnotetext{
${ }^{8}$ Sogenannte Geschlechtstypisierung (mehr dazu in Allport 1971).

${ }^{9}$ Popular crowd is a significant mass of people who gain visibility and status in the [peer] cohort [Eckert 2014:251].
} 


\subsection{Die mit der Geschlechtsidentität verbundenen Stereotype}

Die Geschlechtsidentität ist mit der Bezugnahme eines Individuums auf seine Geschlechterklasse und mit seiner Beurteilung hinsichtlich der Idealvorstellung von Männlichkeit/Weiblichkeit verbunden. In der Entwicklung der Geschlechtsidentität werden einige Stadien der Geschlechteridentifikation unterschieden - die sog. „grundlegende“ Geschlechtsidentität, die bis zum Alter 3 Jahre herausgebildet wird und die Selbstwahrnehmung des Kindes $\mathrm{zu}$ diesem oder jenem Geschlecht und die weitere Wahrnehmung der Geschlechterzugehörigkeit als konstante Größe voraussetzt. Die Identifizierung fordert entsprechendes Verhalten zur Bestätigung eigener Geschlechterzugehörigkeit und setzt die Aneignung von Stereotypen voraus, die den individuellen Vorstellungen über das Männliche/Weibliche entsprechen. Die erworbenen stereotypen Vorstellungen wirken ,verhaltenssteuernd, weil sie Wissen über geschlechtsangemessenes Verhalten in einer bestimmten Situation oder gegenüber bestimmten Personen bereitstellen“ (Gottburgsen 2000: 74). Im Schulalter wird die Geschlechtsidentität bei Jugendlichen infolge der Interaktion mit Schulkameraden konstruiert und zeichnet sich durch „einen gewissen Anpassungsdruck aus, welcher besonders deutlich wird, wenn Mädchen oder Jungen gegen geschlechtsspezifische Normen verstoßen“ (Schmalzhaf-Larsen 2004: 41).

Insgesamt betrifft die geschlechtsspezifische Stereotypisierung die physischen, psychologischen und kognitiven Eigenschaften und Besonderheiten des Sprachgebrauchs beider Geschlechter. So werden die physischen Charakteristika mit der körperlichen Stärke des Mannes assoziiert - ,physical masculinity, associated with physical power“ (Eckes/McConnell-Ginet 2006: 47), die Schwäche gilt als typisch weibliches Merkmal.

Die zugeschriebenen geschlechtsspezifischen psychologischen Charakteristika beruhen z. T. auf der Rollenverteilung - traditionelle weibliche soziale Rollen liegen in der privaten Sphäre (Haushalt, Familie) und werden mit der Mutterrolle assoziiert, die männlichen dagegen - mit mehr Aktivitäten in der Gesellschaft und mit der Familienversorgung. So erscheinen die Frauen als taktvoll, zart, weich, schutzbedürftig, freundlich und nett (cf. Samel 2000; Basow 1992; Mills 2004). Den Männern werden solche Charaktereigenschaften wie Aktivität, Abenteuerlust, Direktheit, Ehrgeiz, Objektivität, Freude am Wettbewerb und Konkurrenz zugeschrieben. Die aufgezählten Charaktereigenschaften bestimmen auch gewissermaßen den Status des Mannes innerhalb einer sozialen Gruppe und bilden Modelle der Selbstidentifikation. Z. B. kann das ideale männliche Modell der Selbstidentifikation in vier Imperativen der Männlichkeit erklärt werden (Badinter 1993: 160-161):

- no Sissy stuff (nichts Weibliches) - der Imperativ fordert vom Mann Stoizismus (kein Klagen) - „ein wirklicher Mann ist bar jeder Weiblichkeit“;

- the big wheel (eine wichtige Persönlichkeit) - Männlichkeit wird an der Elle des Erfolgs, der Macht und der Bewunderung, die einem entgegengebracht wird, gemessen;

- $\quad$ the sturdy oak (die feste Eiche) - rückt die Notwendigkeit, unabhängig zu sein und sich nur auf sich selber zu verlassen, in den Vordergrund;

- give'em Hell (zum Teufel mit allen) - das Imperativ betont die Verpflichtung, sich als stärker zu erweisen als die anderen, notfalls mittels Gewalt. 
Noch ein wichtiges Prinzip für die Herausbildung der Männervorstellung ist „nicht fügsam, abhängig, unterwürfig hinsichtlich seiner körperlichen Erscheinung und in seinem Verhalten nicht feminin zu sein“ (ibd.: 143).

Als ursprünglich männliche Präferenz gilt das Interesse an Naturwissenschaften und Technik (,technical maskulinity of upper-middle class“ (Eckert/McConnell-Ginet 2006: 47), zur weiblichen Interessensphäre werden Geistes- und Sozialwissenschaften gezählt. Als typische Vorlieben des weiblichen Geschlechts gelten Mode und Shopping. Außerdem wird das weibliche Sprachverhalten als weniger aggressiv, emotional, grammatisch korrekt, höflich und weniger präzise beurteilt. Davon ausgehend weisen die Sprachforscher (cf. Tannen 2005:292) auch auf den Unterschied zwischen weiblichem und männlichem Kommunikationsstil hin, der vereinfacht als binäre Opposition verstanden werden kann (Hierarchie- und Statusbewahrung im Gespräch mittels Konflikte, Übernahme der Erzählerrolle; Kommunikation in der Öffentlichkeit vs. Übernahme der Hörerrolle; Kommunikation in privater Sphäre; Vermeidung der offenen Konfliktsituation bei Frauen).

Die Geschlechterstereotype sind aber heterogene Gebilde, die eine Vielzahl von Stereotypen/Substereotypen einschließen und in verschiedenen Bereichen der menschlichen Tätigkeit vertreten sind. Zusammenfassend lässt sich sagen, dass die Geschlechterstereotype folgendermaßen klassifiziert werden können:

- abhängig davon, ob die Stereotype schematische Vorstellungen über Vertreter des eigenen oder anderen Geschlechts präsentieren, kann man von oft positiv/negativ beladenen geschlechterbedingten Auto- und Heterostereotypen sprechen;

- Geschlechterstereotype, die sich auf verschieden Sprachniveaus manifestieren können (bsw. in der Wortsemantik, phraseologischen Einheiten, Syntagmen/Sätzen).

Die Geschlechterstereotype sind also als komplexe kognitiv-kulturelle Strukturen des Bewusstseins $\mathrm{zu}$ verstehen, die oft vereinfachte Vorstellungen über Eigenschaften und Verhaltensweisen, Familien- und Berufsrollen des Menschen einschließen und in sprachlicher und nichtsprachlicher Form vertreten sein können.

\section{Die Geschlechterstereotype in der Substandardmetapher}

Die Geschlechterstereotype können sich in der sprachlichen Form manifestieren, vor allem auf der lexikalischen Ebene in metaphorischer Form. Anders gesagt bilden solche Gedankenstrukturen wie Stereotype „eine Basis für die Metaphorisierung, weil die jeweiligen Übertragungsmotive mehr oder weniger stark stereotypisiert sind“ (Strauß/Haß/Harras 1991: 148). Der metaphorische Wortschatz der deutschen Jugendsprache, bei dem sich ein großer Teil der Lexeme auf den Mann oder die Frau bezieht, hat als Basis entsprechende Stereotype und verweist auf das Schema „Person eines bestimmten Geschlechts (X) weist die Eigenschaft (Y) auf".

Die geschlechtsmarkierte Substandardmetapher kann vom Standpunkt der kognitiven Linguistik aus auch als Denkstruktur betrachtet werden, die aus der menschlichen Wissensprojektion zwischen Ursprungsbereich und Zielbereich resultiert und ,uns eine Begriffsdomäne durch Rückgriff auf einen anderen Erfahrungsbereich begreifen lässt" (Jäkel 2003: 16). Dabei werden ,jedoch keine isolierten Einzelteile des menschlichen Wissens, 

deutschen Jugendsprache

sondern ganzheitliche Wissensbestände projiziert" (Drewer 2003: 6). Die Projektion von bestimmten Merkmalen des Herkunftsbereichs auf den Zielbereich wird durch Vorstellungen und „das ganze System von national-kulturellen Werten und Stereotypen“ (cf. Telija 1998: 40) bestimmt, die infolge der menschlichen Interaktion mit der umgebenden Welt entstanden sind. So resultiert bsw. die gastronomische Substandardmetapher aus der Verbindung der Herkunftsdomäne „Mensch“ mit der Zieldomäne „Essen“.

\begin{tabular}{|c|c|c|c|}
\hline Ausgangsbereich & Mapping-Eigenschaft & Zielbereich & Beispiel \\
\hline Mensch & Geschmack & Essen & Sahneschnittchen \\
\hline
\end{tabular}

Tabelle 1: Korrelation des Herkunfts- und Zielbereichs in der gastronomischen Metapher

Die metaphorische Projektion, die in diesem Fall die Herkunfts- und Zielsphäre verbindet, hat empirische Basis und wird mit dem Genuss, den der süße Geschmack verschafft, in Zusammenhang gebracht. In der gastronomischen Metapher der Art werden also „subjektive Erfahrung/Urteile mit einer sensomotorischen Erfahrung“ (Lakoff/Johnson 1999: 49) verbunden, was manchen Forschern den Grund dafür gibt, solche Metaphern der primären Metapher VERLANGEN IST HUNGER (Grady 1999: 85) unterzuordnen. In solch einer Metapher hat der Referent (Hunger) nicht die unmittelbare perzeptive Basis, aber er gehört in den Bereich der unmittelbaren menschlichen Erfahrung und geht also aus stereotypen Vorstellungen hervor, die subjektiv und empirisch fundiert sind. Die Basis für die primäre Metapher ist die primäre Szene, die aus der menschlichen Erfahrung erwächst, dass die physische Empfindung des Hungers das Verlangen nach dem Essen voraussetzt. In unserem Fall kann behauptet werden, dass die folgenden Vorstellungen die Basis für eine Bedeutungsübertragung bilden können: Süßigkeiten verschaffen Genuss, wecken das Verlangen sie zu essen und so wird auch der Hunger befriedigt. Bei den gastronomischen Bedeutungsübertragungen nach dem Schema „MENSCH - ESSEN“ (schön ${ }^{10}$ ) wird zugleich das Stereotyp ,alle schönen Menschen (X) seien Objekt der Begierde (Y)“ aktiviert.

Vom semantischen Standpunkt aus, wird der Mechanismus der Metaphernbildung durch Aktualisierung des differentiellen Sems in der Bedeutung des Wortes erklärt, wobei die differentiellen Seme durch Stereotype bedingt werden können. Beispielsweise können bei der Bedeutungsübertragung nach dem Schema Pflanze $\rightarrow$ Frau (Mimose - schüchterne Frau) folgende Seme herausgefiltert werden: Pflanze (Nichtlebewesen); hat die Form eines Strauchs; gelbe Farbe der Blüte; klappt ihre Blätter nach der geringsten Erschütterung abwärts. Das Merkmal der Pflanze die Blätter abwärts $\mathrm{zu}$ klappen ist für die Bedeutungsübertragung entscheidend und liegt auch der stereotypen Vorstellung zugrunde, dass alle schüchternen Frauen zurückhaltend sind.

So sind die geschlechtsmarkierte Substandardmetapher und Geschlechterstereotype unmittelbar verbunden, weil bestimmte Stereotype die Informationen über typisch männliche/weibliche Eigenschaften und Charakteristika enthalten können, die bei der Bedeutungsübertragung in Form von Assoziationen auftreten.

\footnotetext{
${ }^{10}$ Die von den Probanden gegebenen gastronomischen Metaphern bezogen sich vorwiegend auf schöne Frauen.
} 


\section{Datenanalyse}

$\mathrm{Zu}$ den wichtigsten Datentypen, die in diesem Aufsatz genutzt werden, gehören:

- Fragebogenerhebungen an Bildungsinstitutionen, wobei die Probanden nach dem Kenntnis der Substandardmetaphern gefragt wurden;

- Ausgewählte Zeitschriften und Internetquellen, in denen die mittels der Befragung gegebenen Metaphern im Kontext vorkommen. Die Kontextbeispiele wurden ergänzend zur Veranschaulichung einzelner metaphorischer Modelle herangezogen.

Die Analyse von Substandardmetaphern, die infolge der Befragung auf Stimuli Frau/Mann gegeben wurden, erfolgte mittels quantitativer Berechnungen. In der Fragebogenaktion, die vor 2 Jahren in Wuppertal durchgeführt wurde, nahmen insg. 516 Probanden teil, darunter 156 junge Frauen und 306 junge Männer im Schul- und Studentenalter. Das Alter der Befragten lag zwischen 12 bis 30 Jahren, wobei die Mehrheit der Probanden im adoleszenten ${ }^{11}$ Alter (302 TN) war. Es wurden auch 203 Probanden im postadoleszenten Alter und 11 - im präadoleszenten Alter befragt. Das Metaphernkorpus besteht aus 246 Metaphern, die im Rahmen einer Fragebogenaktion zu Stimuli Frau/Mann gegeben wurden. Die Datenverarbeitung hat gezeigt, dass die meisten der von den Probanden angeführten Metaphern auf das andere Geschlecht referiert haben.

Die männlichen Probanden gaben insg. 183 Metaphern an, von denen $142(77,6 \%)$ zur Bezeichnung von Frauen genannt wurden; die weiblichen Probanden gaben insg. 63 Substandardmetaphern an, unter denen 41 (65,1\%) Einheiten auf das männliche Geschlecht referiert haben.

Zur Bezeichnung der Frauen gaben die männlichen Probanden meist die Metaphern an, die das Aussehen der Frauen betrafen (85,9\% oder 122 von 142) und zur Bezeichnung der Männer gaben die männlichen Probanden Substandardmetaphern an, die sich auf deren Verhalten und Charakter bezogen (63,4\% oder 26 von 41). Die Mehrzahl der von den Probandinnen gegebenen Metaphern zur Bezeichnung der Frau bezog sich auf das weibliche Aussehen (77,3\% oder 17 von 22) und der Anteil der Metaphern, die die männlichen Charaktereigenschaften/Verhaltensweisen betrafen, war etwas höher als der zur Bezeichnung des männlichen Aussehens (51,2\% oder 21 von 41).

Alle Substandardmetaphern wurden nach dem gemeinsamen Motivationsmuster in gastronomische, zoomorphe, Artefakten- und andere Metapherntypen unterteilt. Schematisch können die Berechnungen in Form einer Tabelle dargestellt werden.

\begin{tabular}{|c|cc|cc|}
\hline Metapherntyp & \multicolumn{2}{|c|}{ männliche Probanden } & \multicolumn{2}{c|}{ weibliche Probanden } \\
\hline \multirow{2}{*}{ Gastronomische Metapher } & Frequenz & Anteil in \% & Frequenz & Anteil in $\%$ \\
& 32 & 17,4 & 16 & 25,3 \\
\hline
\end{tabular}

\footnotetext{
11 Das adoleszente Jugendalter umfasst die Altersgruppe der 12 bis 19-Jährigen. Die Gliederung des Jugendalters erfolgt nach J. Androutsopoulos (2001).
} 
Oksana Khrystenko: Die Manifestierung von Geschlechterstereotypen in Metaphern der deutschen Jugendsprache

\begin{tabular}{|c|c|c|c|c|}
\hline Zoomorphe Metapher & $\begin{array}{c}\text { Frequenz } \\
58 \\
\end{array}$ & $\begin{array}{c}\text { Anteil in } \% \\
31,6 \\
\end{array}$ & $\begin{array}{c}\text { Frequenz } \\
16 \\
\end{array}$ & $\begin{array}{c}\text { Anteil in \% } \\
25,3\end{array}$ \\
\hline Artefaktenmetapher & $\begin{array}{c}\text { Frequenz } \\
43 \\
\end{array}$ & $\begin{array}{c}\text { Anteil in } \% \\
23,4\end{array}$ & $\begin{array}{c}\text { Frequenz } \\
12 \\
\end{array}$ & $\begin{array}{c}\text { Anteil in \% } \\
19,0\end{array}$ \\
\hline $\begin{array}{c}\text { restliche Metapherntypen } \\
\text { (politische, } \\
\text { anthropozentrische, } \\
\text { Pflanzen- und } \\
\text { Sportmetapher) }\end{array}$ & $\begin{array}{l}\text { Frequenz } \\
\begin{array}{l}50 \\
\text { ins }\end{array}\end{array}$ & $\begin{array}{l}\text { Anteil in \% } \\
27,3 \\
183\end{array}$ & $\begin{array}{l}\text { Frequenz } \\
\qquad \begin{array}{l}19 \\
\text { ins }\end{array}\end{array}$ & $\begin{array}{l}\text { Anteil in \% } \\
30,1 \\
63\end{array}$ \\
\hline
\end{tabular}

Tabelle 2: Gruppen von Substandardmetaphern nach dem gemeinsamen Motivationsmuster

Für die weitere Analyse der Geschlechterstereotype, die in den Substandardmetaphern zum Ausdruck kommen, wurden drei Sphären der Stereotypisierung herausgesondert: Stereotype des Aussehens, Stereotype des Charakters/Verhaltens sowie jene der intellektuellen Charakteristika.

\section{Metaphorische Repräsentation von Stereotypen des Aussehens}

\subsection{Gastronomische Metapher}

Unter einer gastronomischen Metapher wird ein Metapherntyp verstanden, dessen innere Sprachform zum thematischen Bereich Essen gehört und die allgemeinen Benennungen von Essprodukten, deren Geschmackscharakteristika, Qualität oder Kochcharakteristika enthält. Die Domäne Essen kann sich auf die empirische Erfahrung beziehen, welche mit Empfindungen des Hungers und des Dursts verbunden ist. Diese empirische Erfahrung könnte für die Bildung der metaphorischen Modelle „MENSCH ist ESSEN“, aber auch für primäre Metapher „VERLANGEN ist HUNGER“ und „VERLANGEN ist EMOTION“ entscheidend sein. Für die Konnektion beider Domänen in der Strukturmetapher „MENSCH ist ESSEN“ sind folgende Mappingmerkmale relevant:

\begin{tabular}{|c|c|c|c|}
\hline $\begin{array}{c}\text { Ausgangs- } \\
\text { bereich }\end{array}$ & $\begin{array}{c}\text { Mapping- } \\
\text { Eigenschaften }\end{array}$ & Zielbereich & Lexeme \\
\hline Mensch & Geschmack & Essen & $\begin{array}{c}\text { Torte, Praline, Süßhäppchen, } \\
\text { Honigtorte, Sahneschnitte, } \\
\text { Zuckertorte, } \\
\text { Bananenkuchen,Süßkirsche }\end{array}$ \\
\hline Mensch & $\begin{array}{c}\text { Eigenschaften } \\
\text { (saftig, sattmachend) }\end{array}$ & Essen & $\begin{array}{c}\text { Kirsche } \\
\text { Filet, Fickschnitzel/Schnitzel }\end{array}$ \\
\hline Mensch & Form & Essen & Wurst, Presswurst, Bratwurst \\
\hline
\end{tabular}

Tabelle 3: Verbindung zwischen konzeptuellen Domänen in der Strukturmetapher 
Die entscheidende Charakteristik bei der Bedeutungsübertragung ist der Geschmack des Essproduktes - eine Eigenschaft, die auch in der Semantik von einzelnen Lexemen oder im Bestimmungswort mancher Komposita vorhanden ist (Torte - Gegenstand (Essprodukt); aus mehreren Schichten bestehend; hat meist süßen Geschmack; meist in Kreisform). Das Mapping zwischen dem Herkunfts- und Zielbereich hängt mit der Eigenschaft des süßen Geschmacks zusammen, die Empfindung des Vergnügens zu geben (süße Stimme = angenehme Empfindung hervorrufend/mit süßen Reden einlullen=für einen Menschen angenehme, liebliche Rede). So kann ein schöner Mensch wie ein süßes Produkt, ein sinnliches Vergnügen bereiten. Von allen gastronomischen Metaphern, die von männlichen Probanden gegeben wurden, haben 93\% auf die Frau referiert, bei weiblichen Probanden waren es nur 56\% der Metaphern. Manche Metaphern dieser Gruppe (Torte, Kuchen, Praline, (Süß)Kirsche, Filet, Wurst) haben laut Ergebnissen der Befragung ausschließlich auf das weibliche Geschlecht referiert.

Man muss sagen, dass in zusammengesetzten Substandardmetaphern anderer semantischer Typen (anthropozentrische oder Artefaktenmetapher) das Bestimmungswort oft auf die gastronomischen Metaphern verweisen kann (Zuckerpuppe, Zuckerweib). Außerdem bilden viele gastronomische Metaphern eine Basis für affixale Bildungen (Zuckerle, Süße/er). Die metaphorischen Modelle „VERLANGEN ist HUNGER“/,VERLANGEN ist EMOTION“ sind mit der gastronomischen Metapher verbunden, in deren Semantik der gut aussehende Mensch als Objekt des Verlangens dargestellt wird. So verknüpft das Modell „VerlangenHunger" die subjektive und sensomotorische Erfahrung, wobei die subjektive Erfahrung als Reaktion auf einen perzeptiven Stimulus (der süße Geschmack) verstanden werden kann. Der süße Geschmack kann andererseits nicht nur mit der Befriedigung der Begierde, sondern auch mit der positiven Emotion der Genussverschaffung verbunden werden.

Die gastronomische Metapher, die die Bereiche 'Mensch - Essen' miteinander auf Grund der Form/Größe des Produktes verbindet, aktiviert die Stereotype, die mit der vermeintlichen Unattraktivität eines übergewichtigen Menschen verbunden sind:

1.) Kürzlich erschien eine Bekannte von mir auf einer Party mit einem Kleid, das sie wie eine neonpinke Presswurst aussehen ließ (http://www.joy.de/liebe/a-28004)

2.) Bevorzugt begrüßte er Marc und mich morgens gleich nach dem Aufwachen damit. Danach folgte immer ein Lachflash. Seit Neuestem lautet seine Anrede „Du dicke Wurst!“ (http://www.luciemarshall.com/de)

\subsection{Zoomorphe Metapher}

Die zoomorphe Metapher geht von der bewertenden Charakteristik des Menschen mittels Tiergestalt aus, wobei das metaphorische Modell „MENSCH ist TIER“ verwendet wird. In der Jugendsprache wird dieser Metapherntyp für die Charakteristik des menschlichen Aussehens, aber auch Charakters/Verhaltens gebraucht. Die von Probanden gegebenen Tiermetaphern mit dem Bezug auf die männliche oder weibliche Person unterscheiden sich voneinander in ihrer Semantik. So geschieht das metaphorische Mapping in den Tiermetaphern zur Bezeichnung der Frauen aufgrund folgender Similaritätsbeziehungen: kleine Größe und daraus resultierende Harmlosigkeit des Tieres - Maus, Spätzchen/Spatzi, 

deutschen Jugendsprache

Hühnchen, Küken, Chick; Grazie/Anmut - Mieze, Pussy; Unattraktivität - Eule, Schreckeule, Nebelkrähe. Die aufgezählten Tiermetaphern haben ausschließlich auf die Frauen referiert, weil manche Vorstellungen wie bspw. die über die Niedlichkeit und Schutzbedürftigkeit der kleinen Wesen stereotypisch gesehen für das männliche Geschlecht nicht relevant sind. Oft werden die in der Befragung angeführten zoomorphen Metaphern von bestimmten Adjektiven begleitet, welche die Bedeutung von einzelnen Zoomorphismen determinieren können. So kann die zoomorphe Metapher Huhn abhängig vom begleitenden Adjektiv die Frau als Objekt der Begierde oder als begrenzte Person charakterisieren:

3.) ich möchte heut mit Dir allein sein/komm laß es uns heut einfach tun/komm Du verrücktes süßes Huhn (www.songtexte.com/de Räuber)

4.) Hey, was soll das sein, ihr blöden Hühner? (Yam,32/2007).

Die der zoomorphen Metapher vorangehenden metaphorischen Adjektive können auf folgende konzeptuelle Modelle „VERLANGEN ist HUNGER“ und „VERLANGEN ist FEUER“ verweisen. Das Modell „VERLANGEN - FEUER“ ist mit entsprechenden sensomotorischen Empfindungen verbunden und nicht nur im Substandard vorhanden. Dieses metaphorische Modell drückt die emotionalen Zustände des Menschen aus, weil Erregung in der Sprache oft als Wärme, Feuer, Erhöhung der Körpertemperatur konzeptualisiert wird:

5.) vor Begierde brennen; heißes Verlangen; von der Liebe entflammt sein; brennendes Verlangen nach Liebe/ Anerkennung

Deshalb bezeichnet die zoomorphe Metapher in Verbindung mit den Adjektiven heiß/hot die attraktive Frau, die als Objekt der Begierde dargestellt wird:

6.) Die Handlung ist an den Film „Bodyguard“ angelehnt, aber viel cooler! Keine Frage, in wessen Armen die heiße Chick am Ende landet! (Bravo Hip-Hop, 4/2007).

In zusammengesetzten Substandardmetaphern können einige Stereotype zum Vorschein kommen, die mit dem Haupt- und Bestimmungswort verbunden sind. Beispielsweise bei der Bezeichnung Zookrähe werden die Vorstellungen aktiviert, die einerseits vom Aussehen des Tieres bestimmt werden. Andererseits sind sie mit dem Erfahrungskomplex, der mit dem Lexem Zoo zusammenhängt, verbunden:

Source domain: $\quad$ Mensch

Target domain: Tier (Zookrähe)

Primary experience: Eine Krähe im Vergleich zu den Tieren in Zoogehegen ist unauffällig

Die zoomorphe Metapher, die von ProbandInnen zur Bezeichnung der attraktiven Frau gegeben wurde, kann einer analogischen Metapher zur Bezeichnung des attraktiven Mannes gegenübergestellt werden (geiler Hengst/Bock). Diese Bezeichnungen appellieren eher an die aktiven, führenden Eigenschaften des Mannes. Trotzdem gibt es in der Jugendsprache einige Metaphern mit verachtender Konnotation, die die Separierung eines übertrieben gestylten Mannes von anderen Männern betonen (Albino-Äffchen). 


\subsection{Artefaktenmetapher}

In den gegebenen Artefaktenmetaphern wird die gutaussehende Person meistens als Nutzobjekt verstanden (Gerät, Feger), wobei sich das metaphorische Mapping hauptsächlich nach folgenden Eigenschaften/Funktionen des Gegenstandes vollzieht:

- Wärme erzeugen (Ofen/heißer Ofen/Fackel);

- Explosionsfähig/große Geschwindigkeit erzeugend (Bombe, Granate, Ausschuss, Topschuss);

- $\quad$ Spielen/Gegenstand zum Spielen (Püppchen/Puppe, Barbie);

- $\quad$ Schlagen (Vergleich mit einem Gegenstand, der zum Schlagen benutzt werden kann Hammer, Keule).

Die Artefaktenmetaphern der Jugendsprache können u. a. mit konzeptuellen Metaphern wie „Mensch (Frau) ist ein Behälter“, „Wirkung der Emotionen - physischer Kontakt", „Verlangen - Hunger“, „Verlangen - starke physische Empfindungen“ korrelieren.

Das metaphorische Modell „MENSCH ist EIN BEHÄLTER“ war in solchen Bezeichnungen wie Büchse, Konserve/Konservendose/Dose vorhanden und von Probanden beider Geschlechter nur mit Referenz auf Frau gebraucht.

Die konzeptuelle Metapher nach dem Modell „Wirkung der Emotionen - physischer Kontakt“ wird mit der Empfindung des Schlages verbunden. In angeführten Personenbezeichnungen wie Hammer (vgl. auch die anthropozentrische Metapher 'Hammerbraut') kann der Bereich „Wirkung der Emotionen“ mit dem attraktiven Aussehen des Menschen in Zusammenhang gebracht werden.

Die Mehrheit der Artefaktenmetaphern tritt im Zusammenhang mit entsprechenden übertragen gemeinten Adjektiven auf, die ihrerseits auf die metaphorischen Modellen „Verlangen - Hunger“ (süßes Gerät), „Verlangen - starke physische Empfindungen“ verweisen. So verbindet das Modell „Verlangen - starke physische Empfindungen“ Verlangen als starken affektiv-emotionalen Zustand mit Empfindungen der Schärfe oder Wärme. Daher wird die attraktive Frau mit einem scharfen Gegenstand oder einem warmspendenden Gegenstand verglichen und tritt als Objekt der Begierde auf:

- $\quad$ scharf sein auf j-n. (vom heftigen Verlangen erfüllt sein);

- $\quad$ scharf aussehen (attraktiv aussehen);

- $\quad$ scharfe Rakete; heißes Gerät (=Bezeichnung für reizende Frau im Substandard)

Das metaphorische Mapping in der Artefaktenmetapher zur Bezeichnung der unattraktiven Frau geschieht in der Regel nach der gemeinsamen Eigenschaft beider Bereiche (resemblance-based primary metaphor), die auf die Schmutzigkeit/den schlechten Zustand des Gegenstandes referiert. Stereotypisch gesehen wirkt äußerlich ungepflegter Mensch unattraktiv: U-Bahnklo (unsauberer, unordentlicher Raum) - unordentlicher, schlecht aussehender Mensch. 
Die Bedeutungsübertragung nach dem Schema „(unattraktive) Frau - Gegenstand“ bezieht sich auf den sensomotorischen Wahrnehmungsbereich und zwar auf die Eigenschaften, die bei der Projektion involviert sind:

- die Massivität des Gegenstandes, die mit dem Übergewicht der Frau in Zusammenhang gebracht wird (Panzer, Minipanzer, Dreitonner). So existiert in der Vorstellung der Substandard-Nutzer das Stereotyp ,unattraktive Frau ist nicht schlank“;

- die physiologischen Empfindungen, welche aus dem Modell „Emotionen physiologische Empfindungen“ hervorgehen können. Die Unattraktivität des Menschen wird an die Emotion des Ekels gebunden, worauf z. B. das Bestimmungswort in der Metapher Kotzpfosten hinweist. Der emotive Bestandteil ist in manchen anthropozentrischen Metaphern vorhanden, in denen das Bestimmungswort auf Empfindung von Angst hinweist (vgl. die Bestimmungswörter in Metaphern - Gruselute, Horrorbraut).

\subsection{Andere semantische Metapherntypen zur Bezeichnung des menschlichen Ausse- hens}

In den in der Befragung vorkommenden Metapherntypen, die in den Bereich menschliches Aussehen fallen, wird ebenso die Unscheinbarkeit des Menschen stigmatisiert, die u. a. aus der Unattraktivität seines Gesichts oder Körpers resultieren kann. Motivierend für manche Metaphern können die politischen Realien oder das Wissen über Objekte der umgebenden Welt sein. So wird die Vorstellung in der politischen Metapher Hartz 4 und der metaphorischen Ableitung Hartzi in Anlehnung an das Wissen über Arbeitslose, Geringverdiener als Hartz 4-Empfänger herausgebildet und mit der niedrigen Wertung eines weniger attraktiven Aussehens assoziiert. Die Pflanzenenmetapher Mauerblümchen erinnert an die Unauffälligkeit der Blumen auf Mauern, was menschliche Unauffälligkeit impliziert. In den zusammengesetzten Sportmetaphern (Gesichtself/Gesichtselfmeter) kann das Bestimmungswort auf einen Körperteil des Menschen (Gesicht) hinweisen und ist ein Fall der Metonymie. So resultiert die Unattraktivität des Menschen aus der Unattraktivität seines Körperteils (Stereotyp „unattraktive Person hat ein unschönes Gesicht“). In manchen entlehnten anthropozentrischen Metaphern (Killer-attraktiver Mann) wird das Funktionsmerkmal aktualisiert (Killer - Funktion: Menschen töten; vom Aussehen eines Menschen umgehaut werden).

\section{Geschlechtsstereotype in der semantischen Gruppe „Charakter/Verhalten des Menschen“"}

Die metaphorische Bedeutungsübertragung in der Jugendsprache geschieht auf Grund der in der Gesellschaft etablierten Stereotype über typisch männliche/weibliche Charaktereigenschaften oder Verhaltensweisen. Traditionell werden in genderbezogenen Studien die geschlechtsbedingten Verhaltensweisen in binärer Opposition dargestellt stark/schwach, aktiv/passiv, dominant/untergeordnet, schweigsam/lästernd usw.

Von den stereotypen Vorstellungen ausgehend, werden die Substandardmetaphern zur Bezeichnung der männlichen Schwäche und Abhängigkeit negativ konnotiert. Die meisten 
der von Probanden gegebenen Metaphern und metaphorischen Pejorativa dieser semantischen Gruppe wurden Männern zugeordnet. Die meisten Metaphern der semantischen Gruppe „Charakter/Verhalten des Menschen“ hatten eine Zweikomponentenstruktur nach dem Schema: Adj.+N, N+N. In diesen Komposita kann das Grundwort meist auf die Begriffskategorie zurückverweisen (bspw. Kategorie „Tier“ oder „Gegenstand“), während das Bestimmungswort meist auf gängige Stereotype rekurriert.

So weist das Adjektiv in Metaphern und Metonymien nach dem Typ Adj.+N auf solche Eigenschaften wie Weichheit, Schlappheit des Körpergliedes als Attribut der Männlichkeit hin (Weichei, Schlappschwanz, Weichflöte). Das Grundwort in solchen Komposita erfährt eine doppelte Bedeutungsübertragung: eine metaphorische (menschliches Körperteil $\rightarrow$ Artefakt/tierisches Körperteil) und metonymische (Körperteil zur Bezeichnung des Menschen).

Das Bestimmungswort in manchen Kompositummetaphern (bspw. Warmflasche, Warmsocke) kann mit der sensomotorischen Erfahrung des Menschen zusammenhängen und sich in der Gegenüberstellung warm/kalt, hart/weich ausdrücken. Die Bestimmungsworte mit der Bedeutung der Wärme und Weichheit können im Bewusstsein der Substandardnutzer mit der Weiblichkeit assoziiert werden, was für das Verhalten des Mannes nicht typisch ist. So werden diese Nomen in Bezug auf den Mann verächtlich markiert. Die Assoziationen, welche das Bestimmungswort (Frauen-) in manchen Komposita und Ableitungen (Frauenvogel, Frauenversteher) hervorrufen kann, sind ebenso für die negative Konnotation der Lexeme entscheidend und werden mit der vermeintlichen Passivität, Empfindlichkeit, Abhängigkeit, Komfortliebe des Mannes verbunden.

Eine andere Gruppe der zusammengesetzten Metaphern betrifft solch menschliche Eigenschaften wie Gesprächigkeit. Die übertriebene Gesprächigkeit einer Person ist hauptsächlich in metonymischen Bedeutungsübertragungen vertreten, die auf Grund der konzeptuellen Metapher „Mund - Behälter“ entstanden sind: Labertasche, Labersack. Die Artefaktenmetaphern Quasselstrippe oder Kommunikationskeule zur Bezeichnung einer gesprächigen Person beinhalten oft den Vergleich mit der Funktion des Telefonapparates und weisen auf die Situation hin, ,als Telefonistinnen und Hausfrauen das Telefon selbständig und eigenwillig zu nutzen begannen“ (Rösler 2007: 151). Manche Lexeme haben die Befragten beider Geschlechter nur in Bezug auf Frauen verwendet (Quasseltante, Schnatterliese, Labertante), was in die stereotype Vorstellung über weibliche übertriebene Gesprächigkeit und Lästereien passt.

\section{Der Ausdruck der Geschlechtsstereotype im semantischen Bereich „Intellektuelle Charakteristika"}

Nach den Ergebnissen der Befragung wurde die Geschlechtsspezifikation in diesem semantischen Bereich minimal ausgedrückt. Nur vereinzelt gegebene Lexeme wie Blondine/Blonde wiesen nur auf die Frau hin und resultierten aus entsprechenden Vorstellungen über deren intellektuelle Beschränktheit. 


\section{$10 \quad$ Fazit und Überblick}

deutschen Jugendsprache

Es kann festgestellt werden, dass die Mehrheit der Metaphern in der Jugendsprache geschlechtsmarkiert und von entsprechenden stereotypen Vorstellungen determiniert ist.

Die Metaphern des semantischen Bereichs „Aussehen“ haben bei Probanden beider Geschlechter vorwiegend auf die weibliche Person referiert. Die Metaphern des semantischen Bereichs „Charaktereigenschaften/Verhaltensweisen“ bezogen sich vorwiegend auf das männliche Geschlecht.

Der semantische Bereich des Aussehens schließt auch die größte Zahl der Metapherngruppen nach der Gemeinsamkeit des Motivationsmusters der inneren Sprachform (gastronomische, zoomorphe, Sport-, Pflanzen-, Artefaktenmetapher) ein. Diese Metaphern korrespondieren mit solchen konzeptuellen Metaphernmodellen wie „Verlangen - Hunger“, „Verlangen positive Emotionen“, „Verlangen - Feuer“, „Objekt - Behälter“, „Verlangen - physische Empfindungen“.

Die Entsprechung der existierenden stereotypen Vorstellungen über das attraktive Aussehen oder die Abweichung von diesen konnotiert die Substandardmetapher positiv oder negativ. Die vorhandenen Stereotype in der Bedeutung mancher Metaphern weisen u. a. auf folgende Kriterien der weiblichen Attraktivität wie schönes Gesicht, Gepflegtheit und Schlankheit hin. In der semantischen Gruppe „Charaktereigenschaften/Verhaltensbesonderheiten des Menschen“ waren solche ,typisch weiblichen“ Eigenschaften wie Unentschlossenheit, Abhängigkeit und Weichheit in der Mann - Vorstellung stigmatisiert.

Weitere Perspektiven der Untersuchung von Geschlechterstereotypen bei Jugendlichen kann man als interdisziplinär bezeichnen - wichtig ist dabei die Berücksichtigung der psychologischen, kulturologischen, soziologischen Erkenntnisse bei den linguistischen Forschungen. Andererseits setzt die linguistische Erforschung der geschlechterstereotypen Vorstellungen die Untersuchung der Manifestierung von Stereotypen auf den wichtigsten Sprachebenen voraus.

\section{Literatur}

Androutsopoulos, Jannis (2001): „Von fett zu fabelhaft: Jugendsprache in der Sprachbiografie“. OBST. Osnabrücker Beiträge zur Sprachtheorie 62: 55-78.

Alfermann, Dorothee (1996): Geschlechterrollen und geschlechtstypisches Verhalten. Stuttgart/Berlin/Köln: Kohlhammer.

Allport, Gordon (1971): Die Natur des Vorurteils. Köln: Kiepenheuer \& Witsch.

Apresian, Jurij Derenikowitsch (1995): Лексическая семантика [Lexical semantics]. Bd. 1. Moscow: Jazyki russkoj kultury.

Badinter, Elisabeth (1993): XY: Die Identität des Mannes. München/Zürich: Piper.

Bandura, Albert/Bussey, Kay (1984): "Influence of gender constancy and social power of sexlinked modeling". Journal of personality and social psychology 47/6: 1292-1302.

Bartminski, Jerzy (2005): Jazykovoj obraz mira: ocherky po lingwistike [language image of the world]. Moscow: Indrik.

Basow, Susan A. (1992): Gender stereotypes and roles. California: Brooks/Cole.

Busse, Dietrich (2009): Semantik. Paderborn: Fink. 
Drewer, Petra (2003): Die kognitive Metapher als Werkzeug des Denkens. Zur Rolle der Analogie bei der Gewinnung und Vermittlung wissenschaftlicher Erkenntnisse. Tübingen: Narr.

Duden. Das Große Wörterbuch der deutschen Sprache. http://www.duden.de/woerterbuch [30.12.2014].

Eckert, Penelope/McConnell-Ginet, Sally (eds.) (2006): Language and gender. Cambridge: Cambridge University Press.

Eckert, Penelope (2014): "The emergence of adolescent language". In: Kotthoff, Helga/Mertzlufft, Christine (Hrsg.): Jugendsprachen. Stilisierungen, Identitäten, mediale Ressourcen. Frankfurt a. M., Lang: 247-259.

Eckes, Thomas (1997): Geschlechterstereotype: Frau und Mann in sozial-psychologischer Sicht. Pfaffenweiler: Centaurus.

Eckes, Thomas (2010): „Geschlechterstereotype. Von Rollen, Identitäten und Vorurteilen“. In: Becker, Ruth/Kortendiek, Beate (Hrsg.): Handbuch Frauen und Geschlechterforschung: Theorie, Methode, Empirie. Wiesbaden, Springer: 178-190.

Gottburgsen, Anja (2000): Stereotype Muster des sprachlichen doing gender. Eine empirische Untersuchung. Wiesbaden: Verlag für Sozialwissenschaften.

Grady, Joseph (1999): "A typology of motivation for conceptual metaphor. Correlation vs. Resemblance”. In: Gibbs, Raymond/Steen, Gerard (eds.): Metaphor in cognitive linguistics. Selected papers from the 5th Cognitive Linguistics Conference. Amsterdam, Benjamins: 79-100.

Gülich, Elisabeth (1978): „,Was sein muss, muss sein‘. Überlegungen zum Gemeinplatz und seiner Verwendung“. Bielefelder Papiere zur Linguistik und Literaturwissenschaft 7: 1-41.

Jäkel, Olaf (2003): Wie Metaphern Wissen schaffen. Hamburg: Kovač.

Kilian, Jörg (2015): „Von blonden Däninnen aus deutscher Sicht. Nationale Stereotype und didaktische Sprachkritik - ein Zwischenbericht aus einem laufenden Forschungsprojekt“". In: Peschel, Corinna/Runschke, Kerstin (Hrsg.): Sprachvariation und Sprachreflexion in interkulturellen Kontexten. Frankfurt a. M., Lang: 155-181.

King, Vera (2009): Adoleszenz/Jugend und Geschlecht. Weinheim/München: Juventa.

Konerding, Klaus-Peter (2001): „Sprache im Alltag und kognitive Linguistik: Stereotype und schematisiertes Wissen“ In: Lehr, Andrea (Hrsg.): Sprache im Alltag. Berlin/New York, de Gruyter: 151-172.

Lakoff, George/Johnson, Mark (1999): Philosophy in the flesh: The embodied mind and its challenge to western thought. New York: Basic Books.

Lippmann, Walter (1990): Die öffentliche Meinung. Bochum: Brockmeyer.

MacGarty, Craig/Yzerbyt, Vincent Yves/Spears, Russel (2002): "Social, cultural and cognitive factors in stereotype formation”. In: MacGarty, Craig/Yzerbyt, Vincent/Spears, Russel (eds.).: Stereotypes as explanations. The formation of meaningful beliefs about social groups. Cambridge, Cambridge University Press: 1-15.

Mills, Sara (2004): Gender and politeness. Cambridge: Cambridge University Press.

Putnam, Hilary (1979): Die Bedeutung von „Bedeutung “. Frankfurt/Main: Klostermann.

Röser, Jutta (2007): „Wenn das Internet das Zuhause erobert: Dimensionen der Veränderung aus ethnographischer Perspektive“. In: Röser, Jutta (Hrsg.): MedienAlltag: Domestizierungsprozesse alter und neuer Medien. Wiesbaden, Springer: 157-172. 

deutschen Jugendsprache

Samel, Ingrid (2000): Einführung in die feministische Sprachwissenschaft. 2. Überarbeitete und erweiterte Auflage. Berlin: Erich Schmidt.

Schaff Adam (1979): „Theorien des Stereotyps“. Integrale Linguistik. Festschrift für H. Gipper. Philadelphia-Amsterdam, John Benjamins: 295-318.

Schaff, Adam (1980): Stereotypen und das menschliche Handeln. Wien/München/Zürich: Europa.

Schmalzhaf-Larsen, Christa (2004): Geschlechtersozialisation im Kontext. Hamburg: Kovač.

Schmidt, Hans-Jörg (2002): „Konzeptionelle Ansätze IV: Die Stereotypensemantik“. In: Cruse, Alan/Hundsnurscher, Franz/Job, Michael (Hrsg.): Lexikologie. Ein internationales Handbuch zur Natur und Struktur von Wörtern und Wortschätzen. Berlin/New York, de Gruyter: 291-296.

Strauß, Gerhard/Haß, Ulrike/Harras, Gisela (1991): Wortbedeutungen und ihre Darstellung im Wortbestand. Berlin/New York: de Gruyter.

Tannen, Debora (2005): "You just don't understand: Women and men in conversation". In: Kirillina, Alla (ed.): Гендер и язык [Language and gender]. Moscow, Jazyki slav'anskoj kultury: 235-510.

Telija, Veronika Nikolaevna (1988): «Метафора как модель смыслопроизводства и ее экспрессивно-оценочная функция» [Metapher as a model of sense production and its expressive-evaluative function]. In: Telija, Veronika Nikolaevna (ed.): Метафора в языке и тексте [Metaphor in Language and Text]. Moscow, Nauka: 26-52.

Trautner, Hanns-Martin (1997): Lehrbuch der Entwicklungspsychologie. Göttingen: Hogrefe.

Trautner, Hanns-Martin (2007): „Geschlechtskategorien und Identität im Jugendalter“. In: Neuland, Eva (Hrsg.): Jugendsprache - Jugendliteratur - Jugendkultur. Frankfurt a. M., Lang: 29-45.

Quasthoff, Uta (1973): Soziales Vorurteil und Kommunikation - eine sprachwissenschaftliche Analyse des Stereotyps. Ein interdisziplinärer Versuch im Bereich von Linguistik, Sozialwissenschaft und Psychologie. Frankfurt/Main: Athenäum.

Quasthoff, Uta (1987): "Linguistic prejudice/Stereotypes". In: Ammon, Ulrich/Dittmar, Norbert/Mattheier, Klaus (Hrsg.): Sociolinguistics/Soziolinguistik: Ein internationales Handbuch zur Wissenschaft von Sprache und Gesellschaft. Berlin/New York, de Gruyter: 785-799.

van Dijk, Teun Adrianus (1984): Prejudice in discourse. Amsterdam: Benjamins.

Zybatow, Lew (1995): Russisch im Wandel: die russische Sprache seit Perestrojka. Wiesbaden: Harrassowitz. 